\title{
Heart Attack Detection System
}

\author{
Abhimanyu. $\mathbf{H}^{1}$, BijuBalakrishnan ${ }^{2}$ \\ IV CSE, JCT College of Engineering and Technology, Coimbatore, Tamil Nadu, India ${ }^{1}$ \\ Associate Professor, JCT College of Engineering and Technology, Coimbatore, Tamil Nadu, India ${ }^{2}$
}

\begin{abstract}
Heart attack is a major death causing disease that can risk in patients lives. If a person is affected by cardiac arrest then the only option is to give medical treatment as soon as possible. What if the patient is a single resident of a house that there is no one there to help him in case of any emergencies. So what if an android Smartwatch is able to detect the heart rate of a person, detect any abnormalities that are happening with his heart. What if the same watch sends notifying messages to the close personalities of the patient whenever he needs them. We are planning to make this alive.

A system that could read the heart's blood pressure rate, respiratory rate, temperature of the body and the pulse rate simultaneously to analyze the condition of the heart. Notify the patient about his condition. Notify the doctor about his patients condition and notify his children. The system uses Internet of Things to connect and systemize the working of the sensors and the detectors. Actually four types of sensors are being used : they are: pulse rate sensors, blood pressure sensor, respiratory sensor and temperature detector. These sensors and detectors simultaneously work together to monitor the hearts condition. Then the detailed results can be checked by using the mobile application
\end{abstract}

Keywords: Heart attack, Atmega Microcontroller, WiFi module, Internet Of Things (IoT)

\section{INTRODUCTION}

For the last few years a lot of cardiac patients are dying on their way to the hospital and before reaching the hospital. The main reason for this is that a majority of the cardiac patients are of the age 50 and above . Out these about a $75 \%$ of the patients are single residents. The main problem of these patients is that they are all on their own. They got nobody to give them CPR or take them to the nearest hospital. So we have developed a Smartwatch that can analyze the data acquired by the sensors to check the condition of the heart. The IoT technology is being used in almost every aspect of life. From home furnishings to medical field. From automobile technology to agriculture. So nowadays IoT is the working domain of a lot of technically served areas. IoT is simultaneous working of sensors and detectors that automatically generates output according to the dynamically generated inputs. The main significance is the interaction between the device with the data that is been stored and analyzed regularly. The output will be updated to the user through any output device. The main target of this system is to help those who are actually single resident senior citizens .

\section{METHODOLOGY}

STEP 1:Defining strategy: Examining of current devices ,review of objectives and targets ,identification of technical requirements, evaluation of future needs.

STEP2: Planning: Analyzingof data, analyzing the patient's current condition, planning adaptive changes that occur with dynamic errors.

STEP3: Design: Design for the Smartwatch and the mobile application will be identified and implemented.

STEP 4: Testing: Testing of the Smartwatch, testing of the mobile application.

STEP 5: Maintenance: Technical support is available to give continuous site maintenance.

\section{III.PROPOSED SYSTEM}

The proposed system includes the continued monitoring of heart rate of a person and signaling the same patient if he needs any medical assistance. The monitored data of the heart will be uploaded to the mobile application which will directly notify his close personalities and the consulting doctors through a SMS notification message. The device works mainly on sensors and detectors. There are four sensors present in it. They are: Pulse Rate Sensor, Blood Pressure Sensor, Respiratory Sensor And Temperature Detector. We are using four sensors to get efficient and actual results on the heart's condition. In the existing system only two sensors were used and the results it generated were not efficient. They tend to give false results on frequent times .The device was not user friendly and it had more complex design. In our proposed system there are only two modules a hardware device which is the Smartwatch itself and the mobile application. The Smartwatch monitors and analyze the heart's rate at every time intervals and will notified to the mobile application at the very same time. Using the mobile application anyone can simply go through his or her heart's condition without going to the hospital. 


\section{V. MODULE DESCRIPTION}

There are three modules present in the system. They are Data Analyzing, The Smartwatch and the Mobile Application. The detailed explanation of above pages are:

1) Data Analyzing: in this module the different data of the heart at which the patient is affected by a cardiac arrest is stored and analyzed. For example for an average person the heart beat will be 60 to 100 beats per minute but at the time of cardiac arrest it can go high upto 400 to 500 beats per minute. Then data regarding the patient and his consulting doctors will also be stored for the emergency medical assistance. The location details of the doctors and the patient is also analyzed. The details on the close personalities of the patient will also be stored for sending the notifications.The stored data will je analyzed throughly and the system implements a continued working of the sensors along with the given data and the dynamically acquired data. The system checks for any change in the acquired data and the stored data and works along with it.
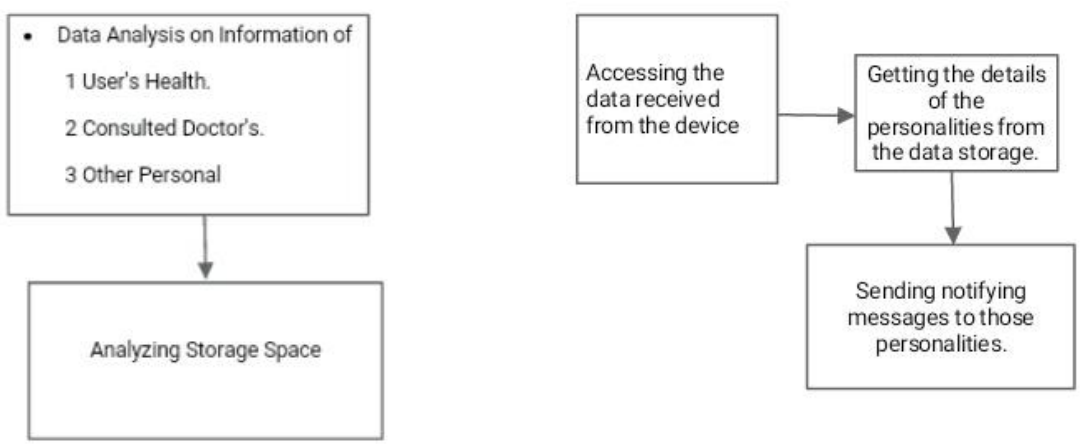

2) The Device: The device or the watch consists of a combination of sensors and detectors working together in the basis of the given and analyzed data and with the data that has been acquired from the device. Then the acquired data will be compared to with the data that has been stored in the device and will be giving a detailed result on the heart's condition. All the data will be stored and updated dynamically on time to time. The device will give signal to the desired person whose contact has been stored in the system already. At anytime of unfamiliarity the device detects it and it will automatically sends the notification messages to the saved contacts. Thedevice consists of an Adruino Compiler, aWiFi module to connect with the mobile. It also consists of sensors and detectors for continuous monitoring of the heart rate.
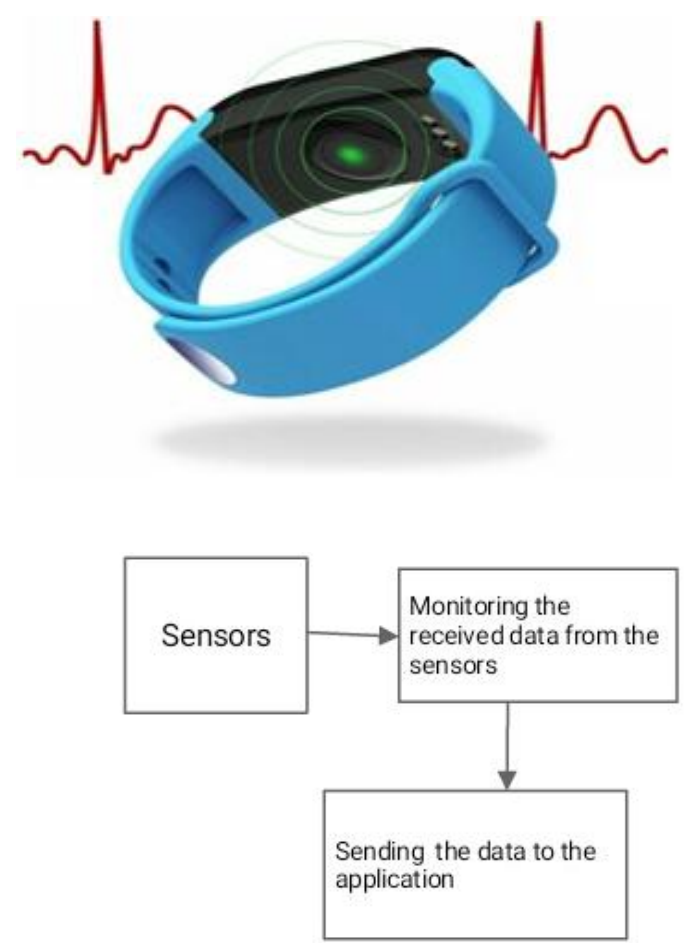


\author{
Vol. 8, Issue 2, February 2019
}

3) The Mobile Application: The mobile application recievesthe data acquired from the device and it will compare the data that is stored in and will generate a test result that will be updated to the application . Then application will be running on android platform. This will make it more user friendly. It lets you an smooth interaction with the device.You will be able to contact with your doctor and can easily change his accommodation at any time. You can easily add up the details of the ambulance driver and the location of the residency should be shared with the diver. So at anytime if you notify through the application the diver can easily find the patient's house. The mobile application is very easy to control. Data accessing and data storing is also made very user friendly so that any person can easily handle the application.

\title{
V.CONCLUSION
}

The proposed system is heart attack monitoring and detecting system. Actually $45 \%$ of the total death is caused by cardiac arrest. Out of this about $60 \%$ of the cardiac patients are above the age of 50 and living as single resident. So at any unfamiliarityis faced by a patient our system detects it and automatically sends notifying messages to the closed person's contacts including his family, doctors and the ambulance driver. It reads the various attributes of a person's heart and give a detailed output on the condition of his heart. The output include the respiratory rate, blood pressure rate, temperature andpulse rate of the person and detailed description about his heart's condition. When he is bad condition and he immediately needs medical attention, then the system automatically allocates an ambulance for the patient. This system can save a lot of senior citizens who are living as single resident. For them at time of cardiac arrest it will be impossible to tell anyone that they have this kind of situation so by this Smartwatch will detect and send the notifying messages in seconds of time. So by anything serious happens he or she can be given proper medical attention and his or her life can be saved.

\section{FUTURE SCOPE}

The system I proposed is mainly triggered with IoT technology. The simultaneous working of sensors and detectors along with the data stored and data acquired dynamically by the device that is worn by the user. So it has an deep core connection with medical IoT also. The medical health care domain is always in need of system that could identify the current situation of the patient always $24 / 7$ thus, through proper research and studies deploy a better and often good system can be developed in the future. Hospitals management could use it as tool or system to monitor the current situation of their patients after their exit from the hospital.

\section{REFERENCES}

[1]. Lossless and Lossy Direct Compression Design With Multi-Signal Symptom Detection for Low-Temperature Wearable Devices. authors-ShuYen Lin Department of Electrical Engineering, Yuan Ze University, Taoyuan, Taiwan, Hao-Te Lin Department of Electrical Engineering, Yuan Ze University, Taoyuan, Taiwan, Yu-Yang Lin Department of Electrical Engineering, Yuan Ze University, Taoyuan, Taiwan.

[2]. Using Internet of Things and biosensors technology for health applications.Authors:HodjatHamidi,Department of Information Technology, Faculty of Industrial Engineering, K.N. Toosi University of Technology, Iran Kimia Fazeli,Department of Information Technology, Faculty of Industrial Engineering, K.N. Toosi University of Technology, Iran

[3]. Wearables and Medical Interoperability: The Evolving Frontier Authors: Florence Hudson,Northeast Big Data Innovation Hub at Columbia University.Chris Clark,Synopsys - Software Integrity Group 